\title{
A giant non-functioning pancreatic neuroendocrine carcinoma founded as an incidentaloma successfully treated with pancreatoduodenectomy
}

\author{
Jacek Paszkowski ${ }^{1}$, Adam Bobkiewicz ${ }^{1}$, Józef Świrkowicz' ${ }^{1}$, Łukasz Krokowicz ${ }^{1}$, Jan Majewski², \\ Tomasz Banasiewicz ${ }^{1}$, Michał Drews ${ }^{1}$ \\ ${ }^{1}$ Chair and Department of General, Endocrinological, and Gastroenterological Oncology Surgery, Poznan University \\ of Medical Sciences, Poznan, Poland \\ ${ }^{2}$ Department of Clinical Pathomorphology, Poznan University of Medical Sciences, Poznan, Poland
}

Gastroenterology Rev 2016; 11 (3): 211-213

DOI: $10.5114 / p g .2016 .61643$

\begin{abstract}
Address for correspondence: Jacek Paszkowski MD, Chair and Department of General, Endocrinological, and Gastroenterological Oncology Surgery, Poznan University of Medical Sciences, 39 Przybyszewskiego St, 61-355 Poznan, Poland, phone: +48604493721 , e-mail: japaszek@op.pl
\end{abstract}

Pancreatic neuroendocrine tumours (PTENs) are a heterogeneous group of tumours that develop from neuroendocrine cells of the pancreas [1]. Pancreatic neuroendocrine tumours comprise a rare group of pancreatic tumours and represent about $1-2 \%$ of all tumours developing within this organ [2]. The potential of neuroendocrine cells to produce and secrete peptides and/ or biogenic amines affecting the clinical presentation of the disease thus determines their division into functioning and non-functioning pancreatic neuroendocrine tumours (functioning, F-PTEN and non-functioning, NFPTEN). General accessibility of cross-sectional imaging of the abdomen has caused a significantly increasing rate of incidentally revealed non-functioning pancreatic neuroendocrine tumours in recent years. The group of NF-PTEN represents the majority of neuroendocrine tumours of the pancreas and is estimated at 60-90\% [3]. An asymptomatic pattern of the disease associated with a lack of the peptide and/or biogenic amine productions means that these tumours may present with considerable size at the time of clinical diagnosis.

We present a giant non-functioning neuroendocrine tumour of the pancreas successfully treated surgically. Surgical treatment remains the treatment of choice in this group of patients. Long-term results of surgical treatment are superior to those found in exocrine pancreatic surgery. The overall survival rate is nearly $90 \%$ in 5-year follow-up [4, 5].

A 72-year-old male was admitted to the hospital because of a tumour of the pancreas. Three months prior to the hospital admission an ultrasound scan had been performed regarding asymptomatic abdominal pain of unknown aetiology. The examination had demonstrated a tumour in the head of the pancreas about $11.5 \mathrm{~cm}$ in size. Besides the intermittent nonspecific abdominal pain, patient did not complain of any other disturbances. He suffered from hypertension and chronic obstructive pulmonary disease (COPD) as comorbidities. The patient's past medical history was notable for cholecystectomy and pneumonia a year previously. On admission, his general condition was good. He did not complain of any problems. According to a physical examination, a palpable mass in the epigastric region was revealed. The patient denied weight loss, elevated temperature, jaundice, or problems with bowel movements. Diagnostics were implemented. An abdominal computed tomography (CT) scan and endocrine evaluation was provided. Imaging of abdominal CT scan demonstrated a huge tumour in the right upper quadrant of the abdomen measuring $14 \times 11 \times 10 \mathrm{~cm}$ (Figure 1). The pathological mass demonstrated features of central necrosis and calcifications. The enhancement due to CT scan was weak and non-equally distributed. Both the liver and other abdominal organs did not reveal any pathology. Bile ducts, both extra- and intrahepatic, were not dilated. Computed tomography scan revealed no evidence of invasion or infiltration of adjacent organs. The patient was qualified for surgery. During the laparotomy a tumour located in the head of the pancreas was found. It was a movable tumour with a rich pathological blood plexus in its wall, without evidence of invasion into adjacent organs. It was fully encapsulated. No lymphadenopathy was founded 


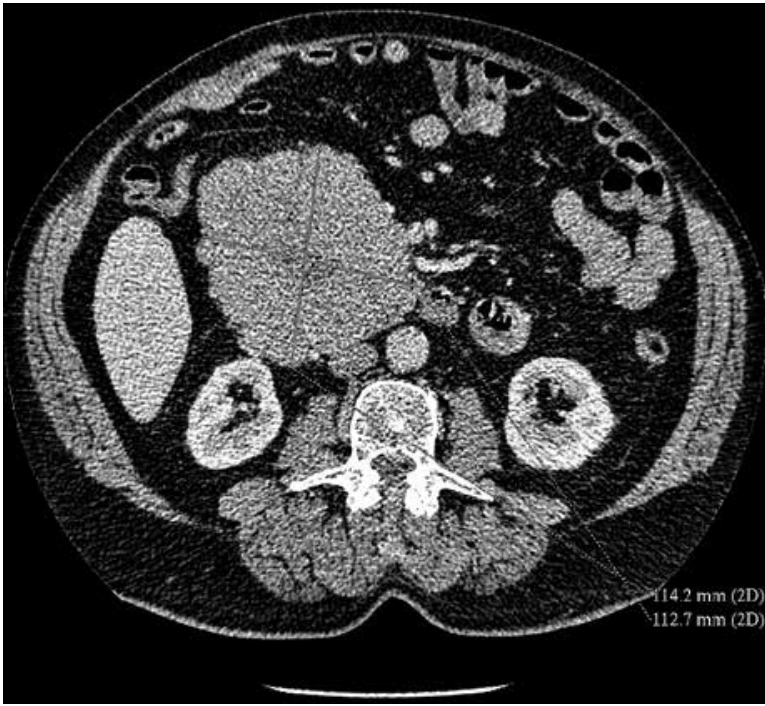

Figure 1. Abdominal CT scan showing a NF-PTEN located in the head of the pancreas. It is important to note that there is no evidence of invasion of adjacent tissue by the tumour

during the surgery. Intraoperatively a specimen of the tumour was taken, which confirmed the neuroendocrine nature of the tumour. Pancreatoduodenectomy was performed (Figure 2). The histopathological report revealed a neuroendocrine tumour of the pancreas. According to WHO 2010 Classification, a neuroendocrine tumour NET G3 was confirmed. All lymph nodes were free from neoplastic cells. Neoplastic infiltration was limited to the head of the pancreas without evidence of infiltration of peripancreatic tissue, duodenum, and stomach. The final pathology did not reveal positive resection margins of the tumour. Immunostaining with chromogranin A and synaptophysin confirmed the neuroendocrine features

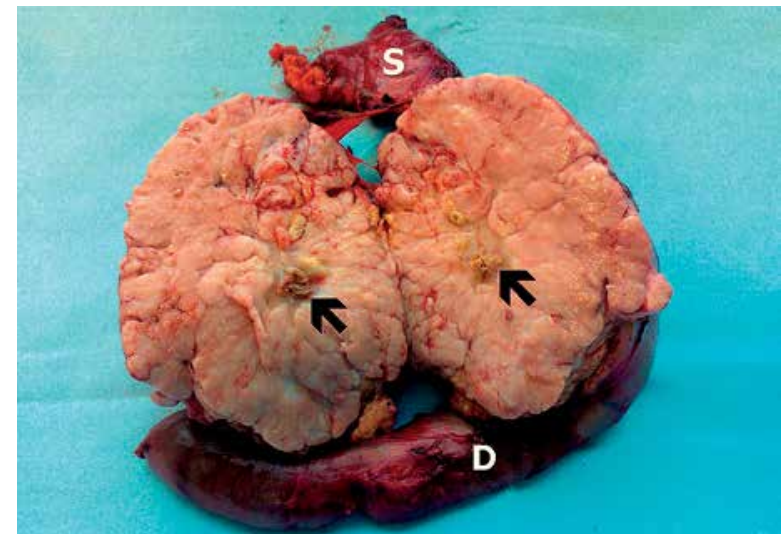

Figure 3. Surgical specimen cut to show some calcification located in the centre of the tumour (black arrows)

$S$-stomach, $D$-duodenum

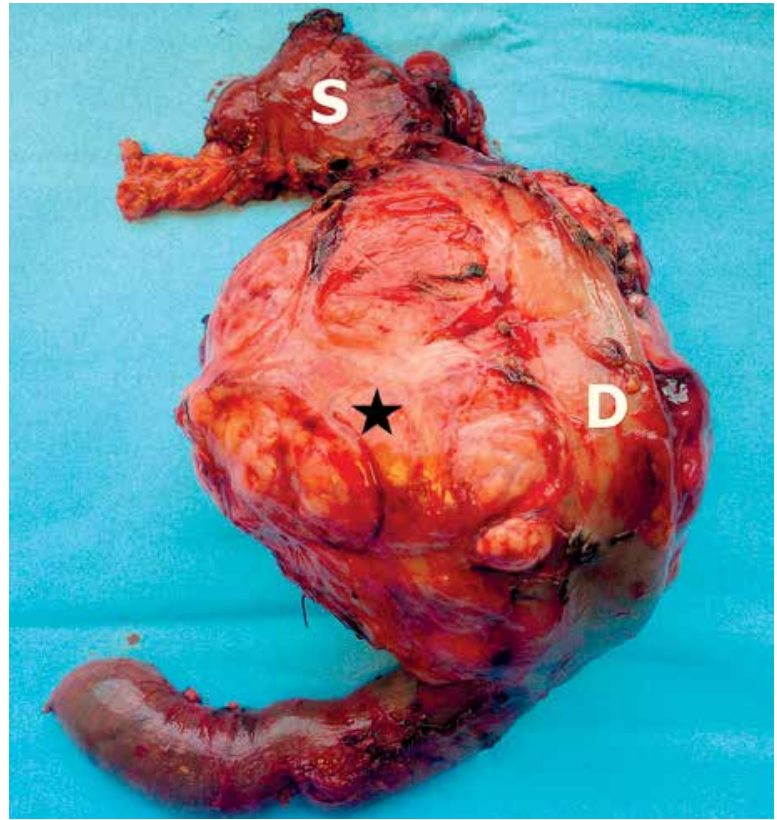

Figure 2. Surgical specimen is comprised of the distal part of the stomach (S), duodenum (D), and the encapsulated mass of the tumour. Note the pathological blood plexus on the tumour $\left(^{*}\right)$. To show the relation between the tumour and the duodenum without any evidence of invasion, the picture is taken from the posterior side of the specimen

of the tumour (Figure 3). Based on mitotic count $<2$ per 10 high-power fields and Ki67 index using MIB1 > 20\% tumour grading G3 was assumed (Figures 4-6).

NF-PTENs are a rare type of tumour of the pancreas. According to the SEER database, NF-PNETs constitute

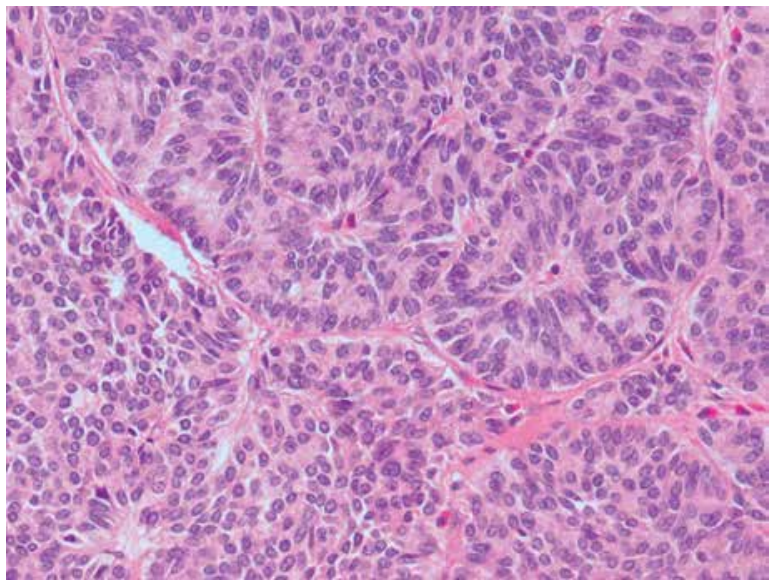

Figure 4. Histopathology presenting poorly differentiated pancreatic neuroendocrine carcinoma (PNEC) $(H+E, 200 x)$ 


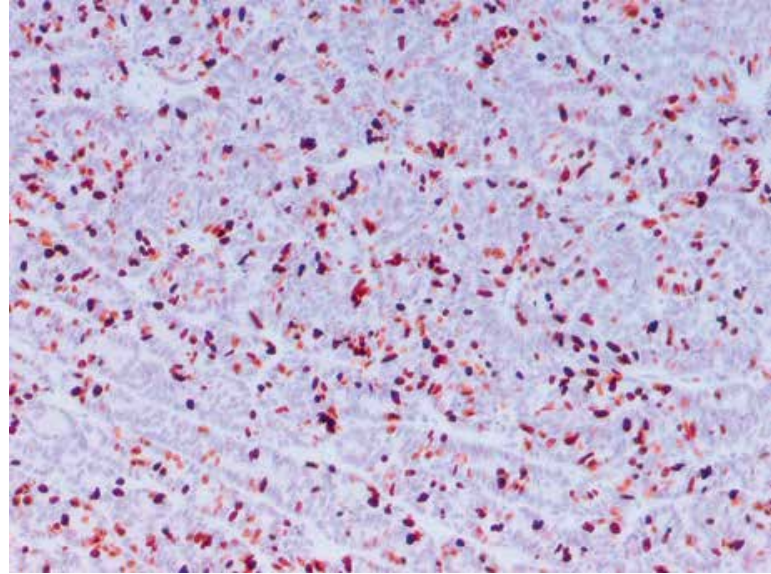

Figure 5. Immunohistochemistry of the specimen showing a strong expression of synaptophysin (40x)

$85 \%$ of all pancreatic neuroendocrine tumours, with the peak incidence in the sixth and seventh decade $[6$, 7]. Widely used medical imaging diagnostics as well as advances in diagnostic tools determine the ability to recognise tumours of the pancreas at an early stage of development, even those without hormonal activity. Rindi et al. proved that as many as half of all pancreatic tumours originally classified as an incidentaloma were finally diagnosed as a NF-PTEN [8]. The symptoms of NF-PTEN are generally non-specific and rarely suggest the diagnosis of pancreatic tumour. Usually such symptoms as abdominal pain, weight loss, or nausea are the first signs of a developing tumour of the pancreas [9]. Despite the improvements as well as the availability of imaging diagnostics, 32-73\% of patients present symptoms of advanced disease with liver metastases at diagnosis [3]. Mostly locally advanced or metastatic NF-PTEN signs are associated with tumour invasion into the surrounding organs causing their dysfunction (cholestasis, bleeding into the peritoneal cavity, bowel obstruction, etc.). Surgery is the treatment of choice in patients with NF-PTEN. Long-term results of surgical treatment of NF-PTEN are characterised by high rates of overall survival and recurrence-free survival. Both of the above-mentioned rates are significantly better in contrast to surgery regarding exocrine pancreatic tumours. Therefore, rational management seems to be the watch and wait strategy associated with rigorous follow-up, which was confirmed in numerous of studies [4].

To the best of our knowledge, the presented report is one of the key NF-PNET cases successfully treated with pancreatoduodenectomy described to date in professional literature. The non-functioning nature of the tumour meant that only nonspecific abdominal pain prompted the patient to seek diagnostics tests.

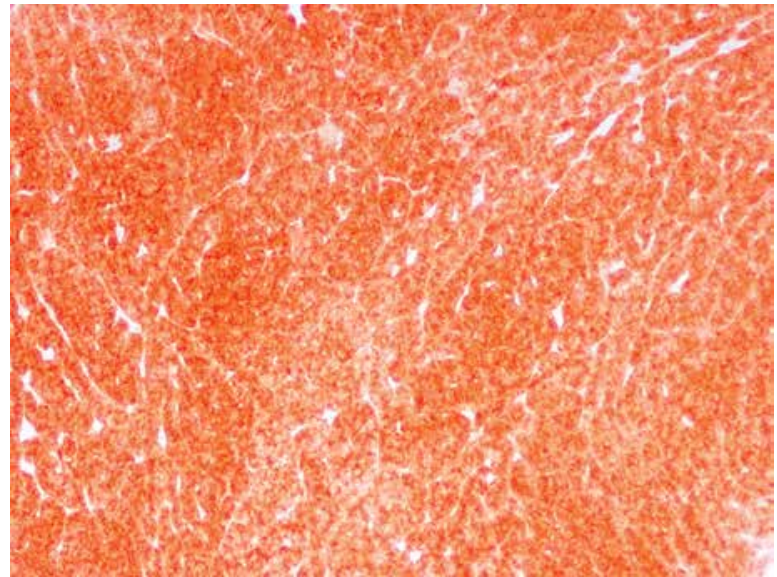

Figure 6 . More than $20 \%$ of the tumour cells are positively stained with MIB1 assessing the proliferation, Ki67 index > 20\% (100x)

\section{Conflict of interest}

The authors declare no conflict of interest.

\section{References}

1. Nakakura EK, Bergsland EK. Islet cell carcinoma: neuroendocrine tumor of the pancreas and periampullary region. Hematol Oncol Clin North Am 2007; 21: 457-73.

2. Oberg K, Eriksson B. Endocrine tumours of the pancreas. Best Pract Res Clin Gastroenterol 2005; 19: 753-81.

3. Falconi M, Bartsch DK, Eriksson B, et al. ENETS Consensus Guidelines for the management of patients with digestive neuroendocrine neoplasms of the digestive system: well-differentiated pancreatic non-functioning tumors. Neuroendocrinology 2012; 95: 120-34.

4. Birnbaum DJ, Gaujoux S, Cherif R, et al. Sporadic nonfunctioning pancreatic neuroendocrine tumors: prognostic significance of incidental diagnosis. Surgery 2014; 155: 13-21.

5. Kim MJ, Choi DW, Choi SH, et al. Surgical strategies for nonfunctioning pancreatic neuroendocrine tumours. Br J Surg 2012; 99: 1562-8.

6. Bilimoria KY, Tomlinson JS, Merkow RP, et al. Clinicopathologic features and treatment trends of pancreatic neuroendocrine tumors: analysis of 9,821 patients. J Gastrointest Surg 2007; 11: 1460-7.

7. Yao JC, Hassan M, Phan A, et al. One hundred years after 'carcinoid': epidemiology of prognostic factors for neuroendocrine tumors in 35,825 cases in the United States. I Clin Oncol 2008; 26: 3063-72.

8. Rindi G, Kloppel G, Alhman H, et al. TNM staging of foregut (neuro) endocrine tumors: a consensus proposal including a grading system. Virchows Arch 2006; 449: 395-401.

9. O'Grady HL, Conlon KC. Pancreatic neuroendocrine tumours. Eur J Surg Oncol 2008; 34: 324-32.

Received: 4.02.2015

Accepted: 11.08 .2015 